\title{
Favorable tumor biology in locally advanced pancreatic cancer- beyond CA19-9
}

\author{
Benedict Kinny-Köster ${ }^{1}$, Joseph R. Habib ${ }^{1}$, Christopher L. Wolfgang ${ }^{2}$, Jin He $^{1}$, Ammar A. Javed $^{1}$ \\ ${ }^{1}$ Department of Surgery, Johns Hopkins University School of Medicine, Baltimore, MD, USA; ${ }^{2}$ Department of Surgery, NYU Grossman School of \\ Medicine, New York, NY, USA \\ Contributions: (I) Conception and design: B Kinny-Köster, JR Habib, AA Javed; (II) Administrative support: None; (III) Provision of study materials \\ or patients: None; (IV) Collection and assembly of data: None; (V) Data analysis and interpretation: None; (VI) Manuscript writing: All authors; (VII) \\ Final approval of manuscript: All authors. \\ Correspondence to: Ammar A. Javed, MD; Jin He, MD, PhD. Division of Hepatobiliary and Pancreatic Surgery, Department of Surgery, Johns Hopkins \\ University School of Medicine, 600 N. Wolfe Street, Baltimore, MD 21287, USA. Email: ajaved1@jhmi.edu; jhe11@jhmi.edu.
}

\begin{abstract}
Patients with pancreatic ductal adenocarcinoma (PDAC) are frequently staged as unresectable locally advanced pancreatic cancer (LAPC) at the time of diagnosis. Recently, the administration of multi-agent induction chemotherapy has resulted in treatment response in up to $60 \%$ of these patients rendering their tumors technically resectable. Operative strategies have evolved to allow for successful oncologic resection of LAPC. These technically complex procedures involving vascular resections and reconstructions are now being performed with increasing safety at high-volume centers. However, even after induction therapy and successful resection, disease recurrence sometimes occurs early on, limiting the benefit of resecting the local tumor. Therefore, selection of surgical candidates should factor in each patient's tumor biology which could result in accurate treatment guidance to improve patient outcomes while avoiding overtreatment. Well-informed patient selection is critical to improve outcomes in LAPC. Multidisciplinary teams have to determine the appropriate care for LAPC patients at the time of reevaluation after administration of induction chemotherapy. At this point the concept of favorable $v s$. unfavorable tumor biology becomes highly relevant and having access to biomarkers that are predictive of tumor behavior are of paramount importance. Currently, CA19-9 remains the only clinically utilized biomarker for PDAC, however, its use is limited by factors discussed in this review. While CA19-9 holds value in patient assessment, additional biomarkers are required that could supplement and improve the current ability to classify tumor biology and predict behavior in individual patients. Recent investigations on the use of circulating tumor DNA (ctDNA) and circulating tumor cells (CTCs) using liquid biopsies, as well as patient-derived organoids to characterize tumor biology have shown promise in achieving precise tumor biology-based patient stratification. Serial assessment of these biomarkers throughout therapy could supplement or even replace the anatomic criteria for resectability in the future.
\end{abstract}

Keywords: Pancreatic surgery; induction chemotherapy; neoadjuvant chemotherapy; recurrence; circulating tumor cells (CTCs)

Submitted Oct 07, 2020. Accepted for publication Feb 03, 2021.

doi: 10.21037/jgo-20-426

View this article at: http://dx.doi.org/10.21037/jgo-20-426

\section{Introduction}

Pancreatic ductal adenocarcinoma (PDAC) remains a devastating disease, with an estimated global incidence of approximately 450,000 with a trend towards an increasing incidence $(1,2)$. Despite improvements in operative techniques and development of more effective systemic and locoregional therapies, the outcomes remain dismal with a 5 -year survival of approximately $10 \%$ for all stages at diagnosis combined (1). Poor outcomes in PDAC are driven 
by its asymptomatic nature resulting in diagnosis at a later stage of disease and early propensity for systemic spread.

A complete oncologic resection remains the only curative-intent option for PDAC; however, at diagnosis, only $10-20 \%$ of patients are deemed resectable (no involvement of adjacent vascular structures). Additionally, approximately $50 \%$ of patients present with metastatic disease, while the remaining $30-40 \%$ present with localized disease with vascular involvement. Based on anatomic criteria, patients with vascular involvement can be further divided into borderline resectable pancreatic cancer (BRPC) and locally advanced pancreatic cancer (LAPC), which is considered unresectable. The National Comprehensive Cancer Network (NCCN) precisely defines LAPC in their 2017 guidelines, which are currently utilized clinically, by implementing an anatomic criteria. Herein, LAPC is defined as localized disease with tumor abutment $\left(>180^{\circ}\right.$ contact) of the surrounding celiac or superior mesenteric artery, or mesoportal venous involvement which is considered unreconstructible (3).

With the introduction of multi-agent chemotherapies such as FOLFIRINOX, it became possible to more effectively treat this cohort of patients $(4,5)$. Aimed at potentially resecting disease over the course of treatment, this initiated therapeutic approach is referred to as "induction" chemotherapy, in contrast to neoadjuvant (resectable tumor stages only with definitively planned surgery) or palliative (no surgery intended) treatment (6). If resection can be performed after induction chemotherapy, it is referred to as "conversion" surgery as the tumor stage became resectable even though initially considered unresectable (LAPC). Recent reports have shown that this approach can result in successful resection in some of these patients resulting in improved survival. However, multiple factors need to be considered when treating this cohort of patients. While extensive pancreatic resections with potential venous and/or arterial reconstructions have now become increasingly safe (acceptable perioperative mortality), the morbidity associated with them remains high. Although obtaining a macroscopically margin-free resection is technically complex in LAPC, increasing experience in novel operative strategies at high-volume surgical centers has demonstrated improved long-term oncologic outcomes for carefully selected cases after induction chemotherapy (5-8). Importantly, when treating PDAC both localized and systemic disease needs to be controlled. Emerging literature shows that despite successful resection and frequent administration of systemic therapy, the rate of recurrence remains high with approximately $70 \%$ of patients experiencing recurrence within the first two years and the predominant pattern being systemic $(9,10)$. It can be inferred that PDAC becomes a systemic (micrometastatic) disease early on and disseminated disease results in recurrence even after successful resection (11). Therefore it is imperative that patient selection should be based not only on the feasibility of a successful oncological resection, but also on tumor biology. Currently, limited tools are available to predict patient's specific tumor biology $(12,13)$.

Biomarkers need to be identified that could help inform the process of decision-making when weighing the potential benefits of performing curative-intent therapy against the potential risk of morbidity associated with a complex surgical procedure, especially in PDAC where systemic disease predominantly dictates long-term outcomes. Herein, we outline the prognostic capabilities that are required from a biomarker that could help guide treatment decisions after successful induction chemotherapy in LAPC. We review the utility of CA19-9 which is the most widely used and validated biochemical tumor marker in PDAC aiming to reflect tumor activity as one parameter of its biology. Furthermore, we discuss potential novel biomarkers that could potentially provide more reliable molecular data on tumor biology and are now under translational investigation to better inform the design of individualized treatment plans in the aspired era of precision medicine.

\section{Biomarkers in LAPC to guide management after induction chemotherapy}

\section{Biomarker portrait-what does "favorable tumor biology" mean?}

The designation of favorable $v s$. unfavorable tumor biology is attributed to a patient's oncological outcome that is driven by the underlying PDAC biology. For patients with LAPC who undergo induction chemotherapy without progression of disease, favorable tumor biology would be more specifically characterized by either the absence of early recurrence after conversion surgery, or stable disease if surgery is not performed.

Several tumor characteristics can influence oncological outcomes and thereby contribute to the classification of favorable vs. unfavorable biology. Firstly, the molecular biology of the local tumor is relevant. While multiple wellestablished factors obtained during the histopathological analysis of the surgical specimen are prognostically 
important, these are not known during the preoperative period and therefore a discussion on them is beyond the scope of this review. Contrastingly, the mutational profile of the local tumor (sequencing of FNA specimen) can be studied preoperatively. It has been shown that PDAC harboring SMAD4 mutations are at a higher risk of distant spread of disease (14). Similarly, germline carriers of $B R C A 1 / B R C A 2$ mutations experienced worse outcomes as opposed to patients with a wild-type germline status (15). While the rate of targetable mutations in PDAC remains low, Blair et al. reported that in PDAC patients with germline BRCA1/BRCA2 mutations the administration of platinum-based chemotherapy resulted in a marked improvement of survival. This suggests that genomic profiling of the local tumor can not only inform about the mutations that can be prognostic, but also help identify potential therapies that may be more effective in treating a particular tumor.

Another aspect of tumor biology that could yield important information is the tumor response to chemotherapeutics. Recent reports have demonstrated that CT imaging cannot precisely evaluate local response to systemic therapy, as it lacks sensitivity to differentiate viable tumor from fibrotic scar tissue in response to multi-agent chemotherapy (16). Therefore, alternative biomarkers are required. As discussed earlier, it has been shown that responsiveness of available chemotherapeutics could be dependent on a patient's tumor mutational profile because certain mutations are potentially targetable by specific agents as indicated by The Cancer Genome Atlas data (17). Furthermore, comprehensive gene expression analyses separated up to four biologic subtypes in PDAC which can be categorized into either "classical" or "basallike" $(17,18)$. The patient's primary tumor genome and transcriptome may also provide insights into the tumor behavior when confronted with a selective pressure due to chemotherapeutic agents. Here, a clonal shift characterized by treatment resistance with the potential for acquired abilities to enter the bloodstream [circulating tumor cells (CTCs)] and to disseminate into distant organs as disseminated tumor cells (DTCs) or micrometastasis is of prognostic significance (19). Molecular analyses of this systemic tumor disease fraction, which by principle is involved in driving the unfavorable recurrent disease burden after margin-free resection of the primary tumor, could be even more accurate in estimating the "prospect of oncological success" as these tumor cells (selected by overcoming induction chemotherapy) remain in the body even after resection. Assessment of this liquid component of disease can provide further information that can help assess tumor biology.

Beyond tumor biology, the host's ability to combat (local and systemic) tumor cells via the immune system is also relevant. Efforts to reactivate the immune system against cancer cells with immune-oncologic treatments (e.g., checkpoint inhibitors) in PDAC have been disappointing in the vast majority of trials. This is believed to be the result of a characteristically low immunogenicity in PDAC where a dense fibrotic tumor stroma and tumor microenvironment signals prevent mechanistic and biochemical tumor infiltration by T-cells (20). Except in patients with a high mutational burden, some long-term survivors have benefitted from specifically developed neoantigens as T-cell targets (21-23). Further research is required to investigate if biomarkers relevant to the host immune system can be identified that can inform towards clinical decision making.

Lastly, the period of time between initial diagnosis and sequential patient reevaluations without detectable distant metastases could be a powerful selection parameter of favorable tumor biology, possibly also indicating sufficient treatment response or/and tumor immunogenicity. However, because time itself is critical in tumor evolution which may potentially lead to chemotherapy-resistant clones, the optimal therapeutic window for curative-intent surgery could be lost if relied on too extensively $(24,25)$.

In summary, favorable tumor biology is dependent on both the local and systemic elements of the tumor. It is currently not feasible and would be invasive to obtain serial tissue biopsies after initiation of induction therapy. Therefore, a particularly valuable source of biomarkers that could be informative of tumor biology would be samples obtained non-invasively. Such blood-based markers could help develop liquid biopsies that could provide information on tumor biology at multiple temporal points during patient management. Having access to these data would be helpful to guide patient care and may enable an accurate selection of patients who are most likely to benefit from a curativeintent resection.

\section{The value of CA19-9 in LAPC}

Carbohydrate antigen (CA) 19-9, or sialyl-Lewis ${ }^{2}$, is the most-widely used tumor marker for PDAC, acting as a surrogate of tumor activity and the overall tumor burden (26). However, there are some innate characteristics of CA19-9 that make it less useful for universal assessment 
of tumor biology in PDAC. Firstly, the synthesis of the physiologic Lewis ${ }^{\mathrm{a}}$ glycoprotein and CA19-9, which is a sialylated derivative of Lewis ${ }^{a}$, is dependent on the FUT3 allele and expressed in $90-95 \%$ of humans. In contrast, $5-10 \%$ of patients with absent Lewis glycoprotein expression are classified as Lewis-negative phenotypes $(27,28)$. These characteristics are important for the interpretation of CA19-9 in PDAC given that Lewisnegative patients will not express CA19-9 yielding false negative results (29). Of note, the prevalence of Lewisnegative patients varies across races with up to $22 \%$ of African-Americans being CA19-9 non-expressors (28).

Additionally, while CA19-9 can help discriminate between PDAC and other non-neoplastic benign pancreatic diseases, it can be elevated in other gastrointestinal pathologies, having a sensitivity of $78.2 \%$ and a specificity of $82.8 \%$ (26). Challenges in the interpretation of CA19-9 also arise in patients with concurrent cholestasis or obstructive jaundice resulting from the tumor which can result in elevated CA19-9.

Several single-center studies have investigated the role of CA19-9 in LAPC after administration of chemotherapy. Two reasonable CA19-9 metrics have commonly been investigated when aiming to estimate prognosis: the actual levels of CA 19-9 in reference to a rather arbitrary threshold [e.g., 1,000 U/mL, $400 \mathrm{U} / \mathrm{mL}$, or $37 \mathrm{U} / \mathrm{mL}$ (normalization)] as well as the dynamic change (relative difference in percent or absolute difference) in reference to a valid CA19-9 value at time of diagnosis (e.g., in absence of hyperbilirubinemia). van Veldhuisen et al. investigated the prognostic value of CA19-9 dynamics after three months of induction chemotherapy in 54 LAPC patients. Patients with a $\geq 30 \%$ decrease in CA19-9 had improved overall survival (OS) as compared to patients with less than 30\% decrease or increasing CA19-9 (median OS: $22.4 v s$. 12.7 months, respectively). This cutoff of a $30 \%$ reduction was found to be most accurate when combined with the radiographically determined Response Evaluation Criteria in Solid Tumors (RECIST) to predict the ability to perform conversion surgery (30). Furthermore, as consistent with prior reports, RECIST response did not correlate well with CA19-9 dynamics for RECIST-stable or RECISTprogressive cancers which emphasizes the discrepancy between biologic tumor activity and morphologic changes observed on imaging (16). For patients where operative resection was possible, Strobel et al. defined a post-treatment cutoff of $400 \mathrm{U} / \mathrm{mL}$ and demonstrated significantly improved OS in patients with a CA19-9 of $<400 \mathrm{U} / \mathrm{mL}$ ( $\mathrm{N}=227$ patients, mainly Gemcitabinebased chemotherapy) (31). An updated analysis of the LAPC cohort from the same center focused specifically on CA19-9 levels and included a subgroup analysis on patients receiving FOLFIRINOX induction chemotherapy and subsequent resection $(\mathrm{N}=64)$. In this cohort a posttreatment CA19-9 value $<92 \mathrm{U} / \mathrm{mL}$ showed improved prognosis, but a $32 \%$ change in CA19-9 could not discriminate overall survival (both cutoffs were derived from a c-statistic analyzing post-treatment resectability after exploration) (32). Two recently published studies reported CA19-9 for selected patients in which surgery was performed with arterial resections after chemotherapy. Truty et al. reported normalization of CA19-9 after induction chemotherapy to be correlated with improved postoperative survival. Importantly, the authors observed that post-treatment CA19-9 was only clinically applicable in about $60 \%$ of patients, as $10 \%$ did not express CA19-9 and $30 \%$ of patients had normal CA19-9 values at time of diagnosis (8). In contrast, surprisingly, in a single-center case series by Bachellier et al. primarily included patients with PDAC undergoing arterial resections $(\mathrm{N}=101)$, CA199 normalization was not associated with OS (7).

Further evidence concerning CA19-9 is available from a few case series comprised of LAPC patients who underwent definitive chemoradiation $(33,34)$. Yang et al. analyzed pre- and post-treatment CA19-9 levels in 54 patients and found that post-treatment CA19-9 of $<50 \mathrm{U} / \mathrm{mL}$ (arbitrarily selected cutoff) and $<85.5 \mathrm{U} / \mathrm{mL}$ (median post-treatment value in their cohort) were both able to successfully discriminate patients based on OS showing a superior prognosis for these patients (median survival 11.1 vs. 7.1 months and 10.3 vs. 7.1 months, respectively). When analyzing the dynamic change of CA19-9 between pre- and post-treatment levels, only a $>90 \%$ decrease demonstrated a significant benefit in median OS (16.3 vs. 7.5 months), and had an increased magnitude over absolute posttreatment values (33). In another retrospective study with a comparable number of patients, tumor stage, and type of treatment regimens it was found that a post-treatment CA19-9 below $<100 \mathrm{U} / \mathrm{mL}$ or a $>40 \%$ reduction were both associated with improved OS (34). However, multiple absolute cutoff values that were investigated (defined in increments of 100 , e.g., $200 \mathrm{U} / \mathrm{mL}, 300 \mathrm{U} / \mathrm{mL}$ etc.) and multiple different cutoffs of a percent reduction (e.g., $10 \%, 50 \%)$ reached statistical significance. Interestingly, in this case series a CA19-9 decrease $>90 \%$ vs. $\leq 90 \%$ could not discriminate survival as suggested previously by Yang 
et al. Besides these results, both study cohorts that were treated with chemoradiation and chemotherapy without surgery (importantly, FOLFIRINOX was rarely used) had relatively poor outcomes for LAPC patients compared to reported outcomes which can potentially be achieved for highly-selected patients who undergo conversion surgery after induction multi-agent chemotherapy $(7,8)$. Of note, a recently published study analyzing CA19-9 dynamics in 131 patients with resectable tumor stages who underwent neoadjuvant therapy found that patients with a normalization of CA19-9 demonstrated superior postoperative OS (46 vs. 23 months) (35). In contrast, the relative magnitude of change was not predictive of survival.

In summary, the quality of evidence available on CA19-9 in LAPC patients is relatively poor for the precise evaluation of CA19-9 after induction chemotherapy when aiming to define a clinically applicable cutoff stratifying prognosis. Even though it could be assumed that a more significant reduction in CA19-9 after chemotherapy is associated with improved prognosis, a longer interval between diagnosis and resection (prolonged duration of chemotherapy) in patients who do not experience disease progression could impact the prognostic ability of CA19-9 in these patients. Nevertheless, a general decreasing trend over the course of treatment and lower CA19-9 levels tend to confer a better conditional prognosis with normalization of levels being the most encouraging metric to study. CA19-9 remains the only clinically utilized biomarker for PDAC, and while it holds value in patient assessment, additional biomarkers are required that could supplement it and improve our current ability to classify tumor biology and predict behavior in individual patients.

\section{Candidates of "liquid biopsies" to derive genomic and transcriptomic tumor traits-circulating tumor DNA (ctDNA) and circulating tumor cells (CTCs)}

Recent investigations of blood-based biomarkers have shown promise. In circulation, fragments of nonencapsulated DNA present in blood outside of cells are referred to as circulating free DNA (cfDNA, also described as cell-free DNA). They are hypothesized to chiefly originate from apoptotic or necrotic cells (passive release), or alternatively be even actively transported across cell membranes (36). In patients with cancer, associated mutations of the tumor from specific genomic loci, e.g., from known driver genes, are detectable as circulating tumor DNA (ctDNA) with increasing sensitivity in plasma (minor allele frequency down to $0.01 \%$ ) using innovative next generation sequencing (NGS) technologies (37). About $95 \%$ of PDAC harbor a mutation in the KRAS gene (especially in, but not limited to the hotspot codons 12 and 13), which makes KRAS a promising target for liquid biopsy with high sensitivity to identify pancreatic cancers (38). However, specificity is comparatively lower, because KRAS mutations can also occur in other malignancies (e.g., colorectal or lung cancer), and in pre-malignant lesions such as pancreatic intraepithelial neoplasms (PanIN) or chronic pancreatitis (39).

Due to these genomic characteristics of PDAC, ctDNA exploration of KRAS has received considerable attention for the development of screening tools for early detection. In 2017, Cohen $e t a l$. were able to detect early stage PDAC using a combination of KRAS-mutant ctDNA and four protein biomarkers (CA19-9, carcinoembryonic antigen, hepatocyte growth factor, or osteopontin) with a sensitivity of $64 \%$ and specificity of $99.5 \%$ (40). The sensitivity of KRAS-mutant ctDNA alone in that study was 30\% with the detected specific point mutation being 100\% concordant with the primary tumor. Importantly, sensitivity substantially increased with higher tumor burden, and reached $88 \%$ in patients with metastatic PDAC (41). For patients harboring an LAPC stage, McDuff $e t$ al. measured ctDNA levels of 38 patients after initiation of chemoradiation and prior to resection and found that ctDNA allele frequency was significantly associated with histologic regression grading (overall survival not assessed) (42). These results indicate that not only the qualitative measurement of ctDNA (presence vs. absence) but also a quantitative metric (mutation allele frequency, i.e., the fraction of mutant-KRAS among all circulating free KRASDNA fragments) may be a valuable indicator of tumor biology, and therefore hold value as a biomarker that could help clinical decision making.

Subsequently, Groot et al. preoperatively investigated $K R A S$ ctDNA as a biomarker to detect tumor recurrence in a cohort of 59 patients (spectrum of all PDAC stages, $41 \%$ underwent neoadjuvant chemotherapy before measurement), and 46 of these patients were followed longitudinally. The preoperative detection rate of ctDNA was $21 \%$ in patients after neoadjuvant treatment $v s .69 \%$ in treatment-naïve plasma samples. Interestingly, detectable ctDNA and tumor size were associated with shorter recurrence-free survival (RFS) and OS, while preoperative CA19-9 value $>200 \mathrm{U} / \mathrm{mL}$ was not independently associated 
with RFS and OS. Immediately postoperatively, ctDNA positivity rate and levels (measured in mutant KRAS droplets per 10,000 total genomic droplets) decreased in most initially positively tested patients when compared to the nearest pre-surgical level. For postoperative monitoring of tumor recurrence, $K R A S$ ctDNA had a sensitivity of $90 \%$ and specificity of $88 \%$ and displayed an ability to predict tumor recurrence at a median of 84 days earlier than conventional imaging. Here, KRAS ctDNA was particularly valuable for patients who did not express CA19-9 and in patients with postoperatively constantly low CA19-9 levels. For several patients who underwent subsequently initiated chemotherapy after disease recurrence, ctDNA changes were associated with response on imaging, again indicating its potential property as a biomarker for tumor burden including monitoring of therapies (43). However, in a study by Bernard et al. which evaluated ctDNA in 34 patients before and after administration of chemoradiation for localized PDAC (positivity rate one third in treatment-naïve measurements), ctDNA dynamics were not correlated with tumor progression based on imaging. For the 20/34 patients who developed metastatic disease, the additionally investigated parameter of circulating exosomal DNA demonstrated a superior performance (44). Even though these findings do not necessarily reflect the property of ctDNA to reflect the overall tumor burden, ctDNA alone could be limited for the identification of an unfavorable tumor biology. This could be reasoned in the observation that such an aggressive trait of evolutionary selected subclones cannot be determined alone from the underlying genome (e.g., KRAS point mutations).

Remarkably, the application of ctDNA by liquid biopsy has gained recent momentum as the FDA has approved an assay to identify EGFR mutations in non-small cell lung cancer. It serves as a non-invasive test to detect and track the sensitivity to a tyrosine kinase inhibitor (osimertinib) targeting specific mutated forms of the epithelial growth factor receptor (45). Such developments rely on rapid testing turnover which has become feasible for ctDNA with modern NGS technologies. For effective monitoring, ctDNA could identify the specific windows of opportunity for therapy, whether systemic or surgical. Such a use-case is promising for patients with LAPC.

Besides the emerging analyses of circulating nucleic acids (cfDNA or exosomal DNA of tumors) from liquid biopsy, circulating tumor cells (CTCs) have been identified as another potentially powerful biomarker with its unique capability to unravel transcriptomic cancer phenotypes. Tumor cells can either be mechanistically shed into circulation, or detach biochemically from the epithelial lining of the primary or metastatic cancer lesion (46). This circulatory invasion has been strongly theorized as a dominating mechanism of metastatic spread in numerous cancers, including PDAC. The obtainment of biological properties to separate from cell-adhesion and survive outside of the tumor microenvironment in the blood stream is referred to as the epithelial-mesenchymal transition (47). This reversible state reflects a tumor cell's plasticity to express a different set of genes, providing the tumor with an increased ability to metastasize. Importantly, proteins characteristic for an epithelial or mesenchymal phenotype can be analyzed on CTCs reflecting biologic functions. Common examples for epithelial markers are cytokeratins, E-cadherin, or EpCAM, whereas a mesenchymal gene program is frequently characterized by the expression of vimentin, N-cadherin, Snail, Zeb1, or TWIST (47). Further markers of interest are generally immunologic markers such as the absence of MHC class I molecules or overexpression of PD-L1, as well as markers typically expressed in stemcells such as ALDH.

CTCs have been detected in both peripheral and portal venous blood of patients with PDAC (48-50). Similarly to ctDNA, CTCs could originate from primary or metastatic disease, and can be thought of as a potential representation of systemic tumor burden at a particular point in time. Poruk et al. detected epithelial-CTCs in 39/50 mainly stage I/II patients. This was associated with poorer overall survival. Of these 39 patients, two third additionally had CTCs which expressed both pan-cytokeratin (epithelial marker) and vimentin (mesenchymal marker); classified as transitional CTCs. Whereas the presence of transitional CTCs was not correlated with overall survival, it was predictive of earlier recurrence when compared to transitional-CTC-negative patients (51). In the CLUSTER trial, CTCs were prospectively investigated in 136 PDAC patients preoperatively and longitudinally after resection (all tumor stages). The study confirmed the presence of epithelial and/or transitional CTCs preoperatively in $96 \%$ of patients (neoadjuvant treatment in $42 \%$ of the included patients). When enumerating the total number of CTCs (epithelial and transitional subtype combined), a significant reduction was observed in patients after neoadjuvant treatment (11 vs. 7 CTCs per $\mathrm{mL}$ blood). In multivariable regression analyses performed to predict tumor recurrence within 1 year postoperatively in patients 
after neoadjuvant chemotherapy, a total number of CTCs $>5 / \mathrm{mL}$, epithelial CTCs alone $>5 / \mathrm{mL}$, or the presence of at least one transitional CTC per $\mathrm{mL}$ of blood were independent predictors (52). This finding emphasizes the potential value of CTC detection even in the setting of prior administration of chemotherapy as it can be applied in the setting of LAPC. In addition to protein-based characterization, recent approaches have been successful to sequence the transcriptome of CTCs in 35 PDAC patients (localized and metastatic disease) and resulted in heterogenous RNA expression profiles which were clustered into three different profiles, each of which enriched by a different stem-cell characteristic gene (53). The $40 \%$ of patients with the highest expression of LIN28B in their CTCs, which characterized one of these three gene sets, had a significantly poorer survival compared to the remaining $60 \%$. Subsequently performed mechanistic cell and animal models confirmed LIN28B as a metastatic driver and potential drug target (53). Although CTCs promise exclusive insights into tumor biology, a major challenge is its overall rarity and the technical complexity to detect and enumerate these cells, which is currently not high-throughput for pancreatic cancer (technologies described elsewhere) (48). The Food and Drug Administration (FDA) has approved the high-throughput CellSearch system for CTC detection in metastatic breast, prostate and colorectal cancer (54). This technology filters cells from blood by epithelial markers, and thereby cannot detect the potentially unfavorable mesenchymal CTCs. In a clinical trial utilizing the CellSearch system, CTCs were detectable in only $12 / 211(5.7 \%)$ patients with PDAC or periampullary cancer (55).

Cumulatively, these findings related to ctDNA in PDAC are encouraging to introduce a novel biomarker in particular to reflect overall tumor burden which often becomes systemic at very early stages. CTCs in PDAC to date suggest that these cells could be complementary valuable to provide important knowledge regarding the individual disease biology and the mechanisms for metastasis. For the subset of LAPC patients, comprehensive trials investigating ctDNA or CTCs are currently lacking to precisely classify a patient's tumor biology as favorable for the purpose to inform optimal decision-making.

\section{Patient-derived organoids (PDOs)—an individualized molecular model on the horizon for LAPC prognostication?}

Organoids are three-dimensionally growing cell cultures used as organ-like $e x$ vivo models, which have successfully been derived from cancer cells from surgical specimens or biopsies of patients (56). These patient-derived organoid (PDO) cultures have been demonstrated to be superior in recapitulating the in vivo biology when compared to two-dimensional cell cultures or animal models in pancreatic cancer (57). Furthermore, PDOs have demonstrated to be in high correspondence with the mutational and transcriptomic signature of the human specimen. Therefore, PDAC organoid models are increasingly being studied to simulate chemotherapeutic response, called pharmacotyping, in order to personalize treatment regimens. Tiriac et al. established a PDO library of 66 different molecular signatures reflecting the heterogenous spectrum of PDAC, each with corresponding responsiveness to chemotherapies (58). Recently, Seppälä et al. reported that the turnover to infer results from PDOs who underwent pharmacotyping can be completed within a median of 48 days, bringing PDOs one step closer to practical clinical practice. The success rate of PDO generation for 77 established specimens was $77 \%$ (21\% of patients underwent induction chemotherapy before tissue sampling) and did not differ when established by surgical specimens $v s$. fine-needle biopsies (59).

Although the main clinical application of PDO pharmacotyping is aimed to become a tool to facilitate recommendations for individualized chemotherapy guidance, molecular classification after simulated treatment ex vivo may emerge as another valuable biomarker of the evolutionary selected tumor biology (selective pressure of subclones during chemotherapy). Porter $e t$ al. reported on induced clonal selection of more aggressive tumor cells after the selective pressure of chemotherapy (60). Here certain subclones persisted and survived the chemotherapeutic regimen while more susceptible tumor cells were affected. Further elucidation of this element of tumor biology and how it pertains to minimal residual disease is highly attractive using liquid biopsies and PDOs. However, such a use-case of PDOs in PDAC has not yet been evaluated and would benefit from future research.

\section{Discussion and future directions}

Treatment strategies for patients diagnosed with LAPC are undergoing transformative changes in clinical practice. This is largely credited to improved response rates to multi-agent chemotherapies and, as a consequence, more extensive surgical techniques have emerged to oncologically remove the primary tumor. Complex operative resections 


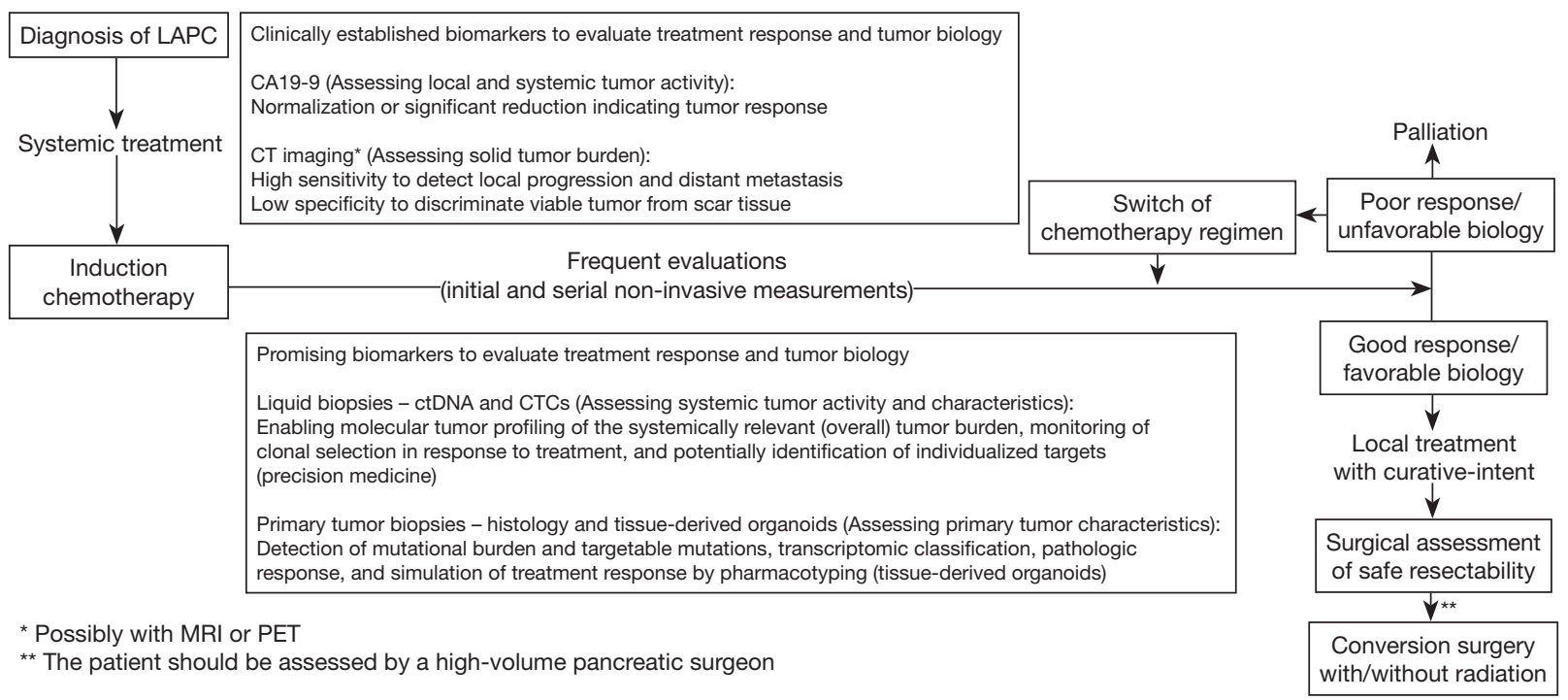

Figure 1 Proposed treatment pathway with integration of biomarkers for a patient diagnosed with LAPC. LAPC, locally advanced pancreatic cancer; CT, computed tomography; MRI, magnetic resonance imaging; PET, positron emission tomography; ctDNA, circulating tumor DNA; CTCs, circulating tumor cells; EUS, endoscopic ultrasound.

are becoming increasingly safe due to innovative techniques and increased surgeon experience in high-volume centers $(5,7,8,61)$. Nevertheless, even following induction chemotherapy and successful operative resection, patients still experience early recurrences mitigating the benefit from these potentially morbid procedures. The poor prognosis has largely been attributed to minimal residual disease and/or clinically undetectable micrometastatic disease in the form of early disseminated tumor cells. Therefore, multidisciplinary teams would fundamentally benefit from precise biomarkers indicating a patient's tumor biology in order to guide treatment recommendations wisely.

In this review, we mainly focus on CA19-9 as the only established PDAC tumor marker and discuss more novel biomarkers including ctDNA and CTCs as innovative parameters that can be studied employing liquid biopsies. We further elaborated on the promising use of molecular tumor data derived by these liquid biopsies and from patient-derived organoids as potential future candidates. When evaluating available data to inform the treatment decisions in the described setting, it is important to select data which were specifically collected for the subset of LAPC patients. Current evidence of studies which include patients with a variety of tumor stages and treatment settings is conflicting. However, prognostic validity of biomarkers may be heavily influenced by these conditions. Early stage or metastatic tumors, as well as treatment-naïve measured parameters may not reflect the expected biologic behavior of LAPC appropriately after induction chemotherapy. Accordingly, a biomarker should assess the dynamic tumor burden and factors linked to chemotherapeutic response. Importantly, available data for the reviewed biomarkers are overall rare in the LAPC cohort, and future studies are necessary to precisely determine what we coined the "prospect of oncological success" and heighten a personalized medicine approach. For the integration of genomic and functional tumor characteristics, a combination of complementary parameters could potentially be superior to a single variable alone. A composite test based on multiple biomarkers could provide the opportunity to study tumor biology at multiple time points and aid in making informed decisions regarding each patient's management. We believe that if such a test became available to us, its clinical integration would provide greater information about tumor biology as compared to CA19-9 alone. Utilizing this tool in clinical practice as depicted by Figure 1 would improve patient selection, avoid overtreatment, and result in improved patient outcomes in LAPC. If serial assessments during induction therapy are indicative of a favorable disease biology, an assessment of the local (primary) tumor by a highvolume pancreatic surgeon is warranted. Contrastingly, in the event that these biomarkers demonstrate poor tumor biology, a change in chemotherapeutics or switch to palliative therapy should be considered in a shared decision-making approach with the patient. 
In conclusion, surgery for LAPC needs to be indicated from both the surgical-technical and oncological standpoint. Well-informed patient selection is critical to improve outcomes in LAPC with potentially curative-intent resections after induction chemotherapy, while avoiding overtreatment. Currently, biomarker-based decision making in LAPC is limited to CA19-9. However, biomarkers are under increasing investigation to guide personalized therapy. A combination of blood-based biomarkers (ctDNA, CTCs, proteins) could provide superior prognostic ability as compared to CA19-9 alone. These novel biomarkers have shown promise in achieving precise tumor biology-based patient stratification and their serial assessment across therapy could supplement or even replace the anatomic criteria, in the future, when determining resectability in LAPC (62).

\section{Acknowledgments}

Funding: BK is supported by the German Research Foundation (KI 2437/2-1).

\section{Footnote}

Provenance and Peer Review: This article was commissioned by the Guest Editor (Elena Rangelova) for the series "Surgery for Locally Advanced Pancreatic Cancer" published in Fournal of Gastrointestinal Oncology. The article has undergone external peer review.

Conflicts of Interest: All authors have completed the ICMJE uniform disclosure form (available at http://dx.doi. org/10.21037/jgo-20-426). The series "Surgery for Locally Advanced Pancreatic Cancer" was commissioned by the editorial office without any funding or sponsorship. The authors have no other conflicts of interest to declare.

Ethical Statement: The authors are accountable for all aspects of the work in ensuring that questions related to the accuracy or integrity of any part of the work are appropriately investigated and resolved.

Open Access Statement: This is an Open Access article distributed in accordance with the Creative Commons Attribution-NonCommercial-NoDerivs 4.0 International License (CC BY-NC-ND 4.0), which permits the noncommercial replication and distribution of the article with the strict proviso that no changes or edits are made and the original work is properly cited (including links to both the formal publication through the relevant DOI and the license). See: https://creativecommons.org/licenses/by-nc-nd/4.0/.

\section{References}

1. Siegel RL, Miller KD, Jemal A. Cancer statistics, 2020. CA Cancer J Clin 2020;70:7-30.

2. Bray F, Ferlay J, Soerjomataram I, et al. Global cancer statistics 2018: GLOBOCAN estimates of incidence and mortality worldwide for 36 cancers in 185 countries. CA Cancer J Clin 2018;68:394-424.

3. Tempero MA, Malafa MP, Al-Hawary M, et al. Pancreatic Adenocarcinoma, Version 2.2017, NCCN Clinical Practice Guidelines in Oncology. J Natl Compr Canc Netw 2017;15:1028-61.

4. Conroy T, Hammel P, Hebbar M, et al. FOLFIRINOX or Gemcitabine as Adjuvant Therapy for Pancreatic Cancer. N Engl J Med 2018;379:2395-406.

5. Hackert T, Sachsenmaier M, Hinz U, et al. Locally Advanced Pancreatic Cancer: Neoadjuvant Therapy With Folfirinox Results in Resectability in $60 \%$ of the Patients. Ann Surg 2016;264:457-63.

6. Sadot E, Doussot A, O'Reilly EM, et al. FOLFIRINOX Induction Therapy for Stage 3 Pancreatic Adenocarcinoma. Ann Surg Oncol 2015;22:3512-21.

7. Bachellier P, Addeo P, Faitot F, et al. Pancreatectomy With Arterial Resection for Pancreatic Adenocarcinoma: How Can It Be Done Safely and With Which Outcomes?: A Single Institution's Experience With 118 Patients. Ann Surg 2020;271:932-40.

8. Truty MJ, Colglazier JJ, Mendes BC, et al. En Bloc Celiac Axis Resection for Pancreatic Cancer: Classification of Anatomical Variants Based on Tumor Extent. J Am Coll Surg 2020;231:8-29.

9. Groot VP, Rezaee N, Wu W, et al. Patterns, Timing, and Predictors of Recurrence Following Pancreatectomy for Pancreatic Ductal Adenocarcinoma. Ann Surg 2018;267:936-45.

10. Jones RP, Psarelli EE, Jackson R, et al. Patterns of Recurrence After Resection of Pancreatic Ductal Adenocarcinoma: A Secondary Analysis of the ESPAC-4 Randomized Adjuvant Chemotherapy Trial. JAMA Surg 2019;154:1038-48.

11. Pommier A, Anaparthy N, Memos N, et al. Unresolved endoplasmic reticulum stress engenders immuneresistant, latent pancreatic cancer metastases. Science 2018;360:eaao4908.

12. Habib JR, Kinny-Köster B, van Oosten F, et al. 
Periadventitial dissection of the superior mesenteric artery for locally advanced pancreatic cancer: Surgical planning with the "halo sign" and "string sign." Surgery 2020; S0039-6060(20)30598-5. [Epub ahead of print]. doi: 10.1016/j.surg.2020.08.031.

13. Kinny-Köster B, van Oosten F, Habib JR, et al. Mesoportal bypass, interposition graft, and mesocaval shunt: Surgical strategies to overcome superior mesenteric vein involvement in pancreatic cancer. Surgery 2020;168:1048-55.

14. Iacobuzio-Donahue CA, Fu B, Yachida S, et al. DPC4 Gene Status of the Primary Carcinoma Correlates With Patterns of Failure in Patients With Pancreatic Cancer. J Clin Oncol 2009;27:1806-13.

15. Blair AB, Groot VP, Gemenetzis G, et al. BRCA1/BRCA2 Germline Mutation Carriers and Sporadic Pancreatic Ductal Adenocarcinoma. J Am Coll Surg 2018;226:630-637.e1.

16. Ferrone CR, Marchegiani G, Hong TS, et al. Radiological and surgical implications of neoadjuvant treatment with FOLFIRINOX for locally advanced and borderline resectable pancreatic cancer. Ann Surg 2015;261:12-7.

17. Cancer Genome Atlas Research Network. Cancer Genome Atlas Research Network. Integrated Genomic Characterization of Pancreatic Ductal Adenocarcinoma. Cancer Cell 2017;32:185-203.e13.

18. Collisson EA, Bailey P, Chang DK, et al. Molecular subtypes of pancreatic cancer. Nat Rev Gastroenterol Hepatol 2019;16:207-20.

19. Kulemann B, Rösch S, Seifert S, et al. Pancreatic cancer: Circulating Tumor Cells and Primary Tumors show Heterogeneous KRAS Mutations. Sci Rep 2017;7:4510.

20. Ho WJ, Jaffee EM, Zheng L. The tumour microenvironment in pancreatic cancer - clinical challenges and opportunities. Nat Rev Clin Oncol 2020;17:527-40.

21. Yarchoan M, Hopkins A, Jaffee EM. Tumor Mutational Burden and Response Rate to PD-1 Inhibition. N Engl J Med 2017;377:2500-1.

22. Balachandran VP, Łuksza M, Zhao JN, et al. Identification of unique neoantigen qualities in long-term survivors of pancreatic cancer. Nature 2017;551:512-6.

23. Meng S, Tripathy D, Frenkel EP, et al. Circulating tumor cells in patients with breast cancer dormancy. Clin Cancer Res 2004;10:8152-62.

24. Yu J, Blackford AL, Dal Molin M, et al. Time to progression of pancreatic ductal adenocarcinoma from low-to-high tumour stages. Gut 2015;64:1783-9.

25. Yachida S, Jones S, Bozic I, et al. Distant metastasis occurs late during the genetic evolution of pancreatic cancer. Nature 2010;467:1114-7.
26. Poruk KE, Gay DZ, Brown K, et al. The clinical utility of CA 19-9 in pancreatic adenocarcinoma: diagnostic and prognostic updates. Curr Mol Med 2013;13:340-51.

27. Westhoff CM, Shaz BH. Chapter 27 - Lewis, I, P1PK and GLOB Blood Group Systems. In: Shaz BH, Hillyer CD, Roshal M, et al., editors. Transfusion Medicine and Hemostasis (Second Edition). San Diego: Elsevier 2013. p. 171-6.

28. Sunwoo HH, Suresh MR. Chapter 9.13 - Cancer Markers. In: Wild D, editor. The Immunoassay Handbook (Fourth Edition). Oxford: Elsevier 2013. p. 833-56.

29. Tempero MA, Uchida E, Takasaki H, et al. Relationship of carbohydrate antigen 19-9 and Lewis antigens in pancreatic cancer. Cancer Res 1987;47:5501-3.

30. van Veldhuisen E, Vogel JA, Klompmaker S, et al. Added value of CA19-9 response in predicting resectability of locally advanced pancreatic cancer following induction chemotherapy. HPB 2018;20:605-11.

31. Strobel O, Berens V, Hinz U, et al. Resection after neoadjuvant therapy for locally advanced, "unresectable" pancreatic cancer. Surgery 2012;152:S33-42.

32. Heger U, Sun H, Hinz U, et al. Induction chemotherapy in pancreatic cancer: CA 19-9 may predict resectability and survival. HPB 2020;22:224-32.

33. Yang GY, Malik NK, Chandrasekhar R, et al. Change in CA 19-9 levels after chemoradiotherapy predicts survival in patients with locally advanced unresectable pancreatic cancer. J Gastrointest Oncol 2013;4:361-9.

34. Koom WS, Seong J, Kim YB, et al. CA 19-9 as a predictor for response and survival in advanced pancreatic cancer patients treated with chemoradiotherapy. Int J Radiat Oncol Biol Phys 2009;73:1148-54.

35. Tsai S, George B, Wittmann D, et al. Importance of Normalization of CA19-9 Levels Following Neoadjuvant Therapy in Patients With Localized Pancreatic Cancer. Ann Surg 2020;271:740-7.

36. Kustanovich A, Schwartz R, Peretz T, et al. Life and death of circulating cell-free DNA. Cancer Biol Ther 2019;20:1057-67.

37. Riva F, Dronov OI, Khomenko DI, et al. Clinical applications of circulating tumor DNA and circulating tumor cells in pancreatic cancer. Mol Oncol 2016;10:481-93.

38. Habib JR, Yin L, Yu J. Pancreatic ductal adenocarcinoma: the role of circulating tumor DNA. J Pancreatol 2019;2:72-5.

39. Maire F, Micard S, Hammel P, et al. Differential diagnosis between chronic pancreatitis and pancreatic cancer: value of the detection of KRAS2 mutations in circulating DNA. 
Br J Cancer 2002;87:551-4.

40. Cohen JD, Javed AA, Thoburn C, et al. Combined circulating tumor DNA and protein biomarker-based liquid biopsy for the earlier detection of pancreatic cancers. Proc Natl Acad Sci U S A 2017;114:10202-7.

41. Bettegowda C, Sausen M, Leary RJ, et al. Detection of Circulating Tumor DNA in Early- and Late-Stage Human Malignancies. Sci Transl Med 2014;6:224ra24.

42. McDuff S, Parikh AR, Hazar-Rethinam M, et al. Using circulating tumor DNA (ctDNA) to predict surgical outcome after neoadjuvant chemoradiation for locally advanced pancreatic cancer (LAPC). J Clin Oncol 2018;36:272.

43. Groot VP, Mosier S, Javed AA, et al. Circulating Tumor DNA as a Clinical Test in Resected Pancreatic Cancer. Clin Cancer Res 2019;25:4973-84.

44. Bernard V, Kim DU, San Lucas FA, et al. Circulating Nucleic Acids Are Associated With Outcomes of Patients With Pancreatic Cancer. Gastroenterology 2019;156:108-118.e4.

45. Ramalingam SS, Vansteenkiste J, Planchard D, et al. Overall Survival with Osimertinib in Untreated, EGFR-Mutated Advanced NSCLC. N Engl J Med 2020;382:41-50.

46. Pantel K, Brakenhoff RH, Brandt B. Detection, clinical relevance and specific biological properties of disseminating tumour cells. Nat Rev Cancer 2008;8:329-40.

47. Kalluri R, Weinberg RA. The basics of epithelialmesenchymal transition. J Clin Invest 2009;119:1420-8.

48. Habib JR, Yu J. Circulating tumor cells in pancreatic cancer: a review. J Pancreatol 2019;2:54-9.

49. Liu X, Li C, Li J, et al. Detection of CTCs in portal vein was associated with intrahepatic metastases and prognosis in patients with advanced pancreatic cancer. J Cancer 2018;9:2038-45.

50. Yu J, Gemenetzis G, Kinny-Köster B, et al. Pancreatic circulating tumor cell detection by targeted single-cell nextgeneration sequencing. Cancer Lett 2020;493:245-53.

51. Poruk KE, Valero V, Saunders T, et al. Circulating Tumor Cell Phenotype Predicts Recurrence and Survival in Pancreatic Adenocarcinoma. Ann Surg 2016;264:1073-81.
52. Gemenetzis G, Groot VP, Yu J, et al. Circulating Tumor Cells Dynamics in Pancreatic Adenocarcinoma Correlate With Disease Status: Results of the Prospective CLUSTER Study. Ann Surg 2018;268:408-20.

53. Franses JW, Philipp J, Missios P, et al. Pancreatic circulating tumor cell profiling identifies LIN28B as a metastasis driver and drug target. Nat Commun 2020;11:3303.

54. Available online: https://www.cellsearchctc.com, accessed on 25 Sep 2020.

55. Hugenschmidt H, Labori KJ, Brunborg C, et al. Circulating Tumor Cells are an Independent Predictor of Shorter Survival in Patients Undergoing Resection for Pancreatic and Periampullary Adenocarcinoma. Ann Surg 2020;271:549-58.

56. Clevers H. Modeling Development and Disease with Organoids. Cell 2016;165:1586-97.

57. Boj SF, Hwang CI, Baker LA, et al. Organoid models of human and mouse ductal pancreatic cancer. Cell 2015;160:324-38.

58. Tiriac H, Belleau P, Engle DD, et al. Organoid Profiling Identifies Common Responders to Chemotherapy in Pancreatic Cancer. Cancer Discov 2018;8:1112-29.

59. Seppälä TT, Zimmerman JW, Sereni E, et al. Patientderived Organoid Pharmacotyping is a Clinically Tractable Strategy for Precision Medicine in Pancreatic Cancer. Ann Surg 2020; [Eoub ahead of print]. doi: 10.1097/ SLA.0000000000004200.

60. Porter RL, Magnus NKC, Thapar V, et al. Epithelial to mesenchymal plasticity and differential response to therapies in pancreatic ductal adenocarcinoma. Proc Natl Acad Sci U S A 2019;16:26835-45.

61. Rangelova E, Wefer A, Persson S, et al. Surgery Improves Survival After Neoadjuvant Therapy for Borderline and Locally Advanced Pancreatic Cancer: A Single Institution Experience. Ann Surg 2019; [Eoub ahead of print]. doi: 10.1097/SLA.0000000000003301.

62. Habib JR, Wolfgang CL. Commentary: Anatomic versus biologic resectability: The role of predictive biomarkers in guiding surgical management. Surgery 2020;168:1017-8.
Cite this article as: Kinny-Köster B, Habib JR, Wolfgang CL, He J, Javed AA. Favorable tumor biology in locally advanced pancreatic cancer-beyond CA19-9. J Gastrointest Oncol 2021;12(5):2484-2494. doi: 10.21037/jgo-20-426 